Phosphorus Research Bulletin Vol. 22 (2008) pp. 41-47

\title{
SOLUBILITY OF MONOALKYL PHOPHATE IN WATER IN THE PRESENCE OF ARGININE AND TRITON, AND SOLUBILIZATION OF METHYL YELLOW THROUGH THE MIXED MICELLE
}

\author{
Takeshi Murata, Satoru Ueno, Tomoaki Hino, and Saburo Shimabayashi* \\ (* Corresponding author: saburo@ph.tokushima-u.ac.jp)
}

Faculty of Pharmaceutical Sciences, Division of Physical Pharmacy, The University of Tokushima, Sho-machi 1-78-1, Tokushima 770-8505, Japan

Keywords: monoalkyl phosphate, hydroxyapatite, surface modification, mixed micelle, Triton-X, arginine

\begin{abstract}
Effects of arginine (Arg) and Triton such as Triton-X (TX) and Triton-N (TN) on the solubility of monoalkyl phosphoric acid (MAP) such as dodecyl (DP), myristyl (MP), and cetyl phosphoric acid (CP) in an aqueous phase were studied. Although these MAPs are scarcely soluble in water, their solubility at $70{ }^{\circ} \mathrm{C}$ increased with a concentration of Triton added. The effect of TN was stronger than that of TX. DP became soluble at room temperature when Arg was added up to the mixing ratio of $\mathrm{Arg} / \mathrm{DP}=3$ in mole. $\mathrm{MP}$ and $\mathrm{CP}$ were hardly soluble yet in the presence of Arg alone while these became soluble after adding Triton together with Arg. Their acidic head group was ionized by Arg, resulting in an increase in its affinity for water. DP was soluble just after the ionization while MP and CP were soluble after the ionization and concomitant formation of the mixed micelle with the Triton added. Methyl yellow, barely soluble in water, was solubilized after entrapment into the mixed micelle. Its solubility increased with a concentration and chain length of the MAP added as a solubilizing agent.

(Received April 6, 2008; Accepted April 30, 2008)
\end{abstract}

\section{INTRODUCTION}

Monoalkyl phosphate (MAP) used in the present paper is composed of a long alkyl chain and an orthophosphate group which are bound together through the ester bonding between them. The MAP used here is in the form of a dibasic monoalkyl phosphoric acid. The carbon numbers of the MAP are 12,14 , and 16, which are designated as dodecyl (DP), myristyl (MP), and cetyl phosphoric acid (CP), respectively. It is known that these are easily adsorbed on the surface of nucleus of hydroxyapatite (HAP, $\left.\mathrm{Ca}_{10}\left(\mathrm{PO}_{4}\right)_{6}(\mathrm{OH})_{2}\right)$ and can regulate its crystal growth as a crystal poison after isomorphous substitution of orthophosphate ion on the nucleus with phosphate group of a MAP in an aqueous phase. Basic Arg was used as a solubilizing agent for the acidic MAP, because the MAP in the acid form is hardly soluble in water while it becomes soluble after the neutralization and ionization by $\mathrm{Arg}^{1}$.

Warabino et $a l^{2,3}$ prepared a new type of HAP in the absence of Arg after mixing an aqueous solution of $\mathrm{CaCl}_{2}$ and $\mathrm{Na}_{2} \mathrm{HPO}_{4}$ with a chloroform solution of a MAP. The chloroform solution was emulsified during the strong shaking and stirring, and the MAP thoroughly contacted the surface of the nucleus of HAP formed in an aqueous phase. That is, this HAP was prepared through the phase transfer reaction of the MAP from a chloroform phase to a water phase under strong agitation. Surface of the HAP was modified in part to hydrophobic one after adsorption of the MAP. The HAP, thus prepared, could adsorb various hydrophobic compounds, forming the surface complex between the MAP and adsorbates. There are some literatures ${ }^{4-7}$ by other researchers also discussing the effects of alkyl chains of MAP on the crystal formation and surface modification of HAP by means of various techniques. Oda et al. ${ }^{8}$ studied the adsorption mechanism of nonionic surfactants, Triton-X (TX) and -N (TN), on HAP in the presence and absence of MAP and Arg in an aqueous phase. Although these Tritons are 
inherently non-adsorbable to the surface, they became adsorbable after forming the mixed micelle together with a MAP.

This fact was explained as follows. The mixed micelle was adsorbed by virtue of isomorphous substitution of orthophosphate ion on HAP with a head group of the MAP protruding from the micelle surface, resulting in the adsorption of the mixed micelle in whole. The admicelle and/or hemimicell of Triton and MAP were formed on the surface, to which another hydrophobic compound was captured through hydrophobic interaction. Thus, the surface of the HAP looks like hydrophobic apparently.

Taking into consideration these facts mentioned above, effects of Triton and Arg on the solubility of MAP are discussed in the present paper. Although both Arg and potassium hydroxide $(\mathrm{KOH})$ are base, their effect on solubility of MAP was different. The reason was briefly considered from the viewpoints that the MAP is a weak dibasic acid and has a long alkyl chain in its molecular structure ${ }^{9,10}$. Solubilizing power of the mixed micelle against a water-insoluble dye methyl yellow (MY) was also discussed.

\section{MATERIALS AND METHODS}

Chemical structures and their abbreviations of the reagents used in this study are shown in FIGURE 1. The MAP was a gift from Kao Co. (Tokyo), which is white fine powder. According to the report from the company, its purity is higher than $99 \%$. Other chemicals used were products of Wako Pure Chemicals Co. (Osaka), Nacalai Tesque, Inc. (Kyoto), or Kanto Chemicals Co. (Tokyo). These were used without further purification.

Solubility of a MAP at $70{ }^{\circ} \mathrm{C}$ was determined in the presence of TX or TN but in the absence of Arg, as follows. Known amount of MAP was suspended in water in a test tube and was preheated up to $55-65{ }^{\circ} \mathrm{C}$ to make the MAP particle melt down. The solution was turbid, forming the droplets. It was then heated up to $70{ }^{\circ} \mathrm{C}$, into which known amount of an aqueous solution of Triton (TX or TN) was gradually added under vigorous stirring. When the solution became clear from the turbid emulsion, the titration attained the end point. Relationship between a concentration of the MAP and that of the Triton was obtained at the end point. Before starting the titration, it was confirmed that cloud point of the Triton is higher than $70{ }^{\circ} \mathrm{C}$.

Solubility of a MAP in the presence of Arg was measured at $30{ }^{\circ} \mathrm{C}$ in a similar manner to that mentioned above. The mixing ratio of Arg to a MAP was kept constant at 3:1 in mole during the titration. An aqueous solution of the MAP mixed with Arg was titrated with an aqueous solution of a Triton. The point where the solution becomes clear was assumed the end point of the titration, and the concentrations of the MAP and Triton were recorded.

Surface tension was measured by means of a $\mathrm{Du}$ Nouy surface tensiometer (Shimadzu, Kyoto) at 20 ${ }^{\circ} \mathrm{C}$ as a function of a concentration of TX, where an aqueous solution of the TX was mixed with Arg alone or with Arg and a MAP at various mixing ratios. The concentrations of all these components are known before the measurement.

Solubilized amount of MY into an aqueous solution of the mixed micelle of TX-MAP was determined at $30{ }^{\circ} \mathrm{C}$, where the ratio of Arg to MAP was also kept constant at 3 to 1 . After excess amount of pulverized MY was added to the sample solution, it was incubated under vigorous agitation for 2 days. The supernatant of the suspension, obtained by centrifugation, was diluted with an equivalent volume of ethanol. The MY concentration was determined by an absorptiometry at $418 \mathrm{~nm}$. The relationship between concentrations of TX and MY was obtained at various concentrations of TX, MAP, and Arg. It was confirmed that TX as well as Arg does not interfere with determination of MY.

\section{RESULTS AND DISCUSSION}

\section{(1) Solubility at $70{ }^{\circ} \mathrm{C}$}

Although MAP alone is insoluble in water at room temperature, it was dissolved at elevated temperature such as $70{ }^{\circ} \mathrm{C}$ in the presence of a Triton. FIGURE 2 shows the concentration of Triton required to dissolve an alkyl phosphate (AP, including MAP and DCP) as a function of a concentration of added AP. The concentration of Triton linearly increased with that of AP. Upper area of the straight line is that where the solution was clear, while the lower area that where the solution was 


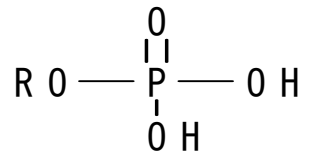

$\begin{array}{lllll}\mathrm{R}=\mathrm{C} & { }_{16} & \mathrm{H}_{33} & \mathrm{CP} \\ \mathrm{R}=\mathrm{C} & { }_{14} & \mathrm{H}_{22} & \mathrm{MP} \\ \mathrm{R}=\mathrm{C} & { }_{12} & \mathrm{H}_{25} & \mathrm{DP}\end{array}$

$\mathrm{CP}$

MP $\quad$ MAP

Monoalkyl phosphate (MAP)

Cetyl phosphate (CP)

Myristyl phosphate (MP)

Dodecyl phosphate (DP)

$\mathrm{R} 0-\stackrel{I_{1}}{\mathrm{P}_{1}}-\mathrm{OH} \quad \mathrm{R}=\mathrm{C} \quad{ }_{0}{ }_{16} \mathrm{H}_{33}$

$\underline{D C P}$

Dicetyl phosphate (DCP)

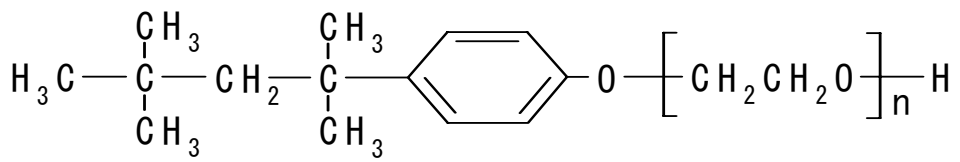

Polyethylene glycol mono- $p$-isooctylphenyl

ether (Triton-X 100, TX), $n \doteqdot 10$

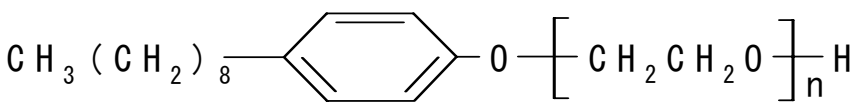

Polyethylene glycol mono- $p$-nonylphenyl

ether (Triton-N, TN), $n \fallingdotseq 10$

$\mathrm{H}_{2} \mathrm{~N}-\stackrel{\mathrm{N}}{\mathrm{N}}-\mathrm{H}_{\mathrm{H}}^{\mathrm{N}}-\left(\mathrm{CH}_{2}\right) \frac{\mathrm{COO}_{3}^{-}}{\mathrm{H}}$

Arginine (Arg)

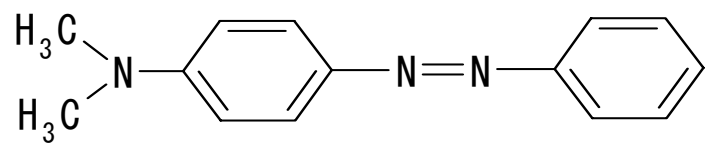

Methyl yellow (MY)

FIGURE 1 Chemical structures and abbreviations of the reagents used in this study. 
turbid, i.e., AP was not thoroughly dissolved. Open symbol with a dotted line shows the data for TN, while closed symbol with a solid line the data for TX.

The results show that TN is stronger than TX in the solubilizing power against AP when compared at the same AP. The slope becomes steeper with the chain length of AP when compared at the same surfactant. These facts are suggesting that AP was solubilized through the intermolecular interaction between hydrophobic chains of the added surfactant and AP. The longer the AP chain becomes, the more amount of the surfactant is required to form mixed micelle and/or to accept the alkyl chain into the micelle. It is well-known that micelle size of a nonionic surfactant becomes larger while crystal and/or solid becomes looser with temperature. Therefore, hydrophobic chains of AP were easily captured into the micelle at high temperature such as $70^{\circ} \mathrm{C}$ although the long chain AP is insoluble in water even in the presence of Triton at room temperature.

The slopes for DCP, shown by two kinds of triangle, are remarkably steeper than those for the MAP. This fact is showing that DCP, which has two alkyl chains, is practically insoluble in water even at $70{ }^{\circ} \mathrm{C}$ in the presence of Triton as a slubilizing agent due mainly to strong intermolecular hydrophobic interaction between DCP itself.

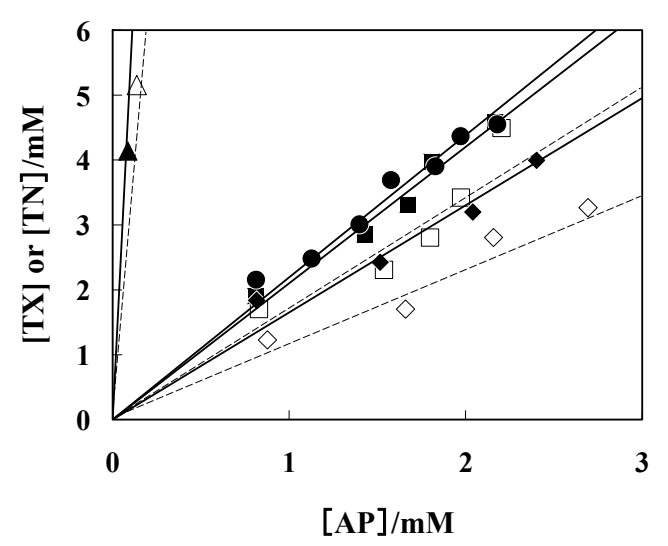

FIGURE 2 Concentrations of TX and TN needed to dissolve an alkyl phosphate (AP) as a function of a concentration of AP added to the test solution at 70 ${ }^{\circ} \mathrm{C}$. Open symbol with dotted line is the data for TN while closed symbol with solid line the data for TX. Symbols: diamond for DP, square for MP, circle for $\mathrm{CP}$, and triangle for DCP. Data for $\mathrm{CP}+\mathrm{TN}$ is not shown here to avoid too much crowd in the figure.

\section{(2) Solubiltity at $30^{\circ} \mathrm{C}$}

DP became soluble in water at $30{ }^{\circ} \mathrm{C}$ when $\mathrm{Arg}$ was mixed at the a molar ratio of $\mathrm{Arg}$ : $\mathrm{DP}=3: 1$. This is because the basic Arg neutralized the acidic head group of DP and the affinity of DP for water increased. MP was almost dissolved in water in the presence of Arg at the same molar ratio of Arg : MP = 3:1, but the solution was still slightly turbid. This fact was explained as follows; because the alkyl chain of MP is longer than that of DP, affinity of MP for water is lower than that of DP and, therefore, the solubility becomes lower. The solution becomes clear, when small amount of TX or TN is added, by virtue of formation of the mixed micelle. In the case of CP, however, the effect of Arg added with a molar ratio of Arg : $\mathrm{CP}=3: 1$ seemed very little. The $\mathrm{CP}$ was practically insoluble in water even in the presence of Arg. Sequence of solubility of the MAP after the addition of Arg was determined as DP, MP, and CP.

Although Arg alone does not accelerate the dissolution of CP, it cooperated with Triton on the dissolution. After dispersing the $\mathrm{CP}$ powder in an aqueous solution of Arg at the mixing ratio of Arg:CP $=3: 1$, the border line was determined by the titration method in a similar manner to that applied to FIGURE 2. The result is shown in FIGURE 3. Data shown by an open circle is that at the constant mixing ratio at $30{ }^{\circ} \mathrm{C}$ while data shown by a closed circle is quoted from FIGURE 2 to compare the effects of Arg and temperature. It is quite interesting that the slope at $30{ }^{\circ} \mathrm{C}$ (open circle) is almost the same as that at 70 ${ }^{\circ} \mathrm{C}$ (closed circle) and, therefore, the concentration of TX to dissolve a given amount of $\mathrm{CP}$ at $30{ }^{\circ} \mathrm{C}$ is almost the same as that at $70{ }^{\circ} \mathrm{C}$. The $\mathrm{CP}$ was dissolved after disintegration of the crystal and entrapment into the micelle at $70{ }^{\circ} \mathrm{C}$, while at $30{ }^{\circ} \mathrm{C}$ it was dissolved after neutralization / ionization of the head group by Arg and capture into the micelle. The effect of adding Arg at $30{ }^{\circ} \mathrm{C}$ is almost equivalent to that of increasing temperature up to $70{ }^{\circ} \mathrm{C}$ from 30 ${ }^{\circ} \mathrm{C}$.

\section{(3) Effect of KOH}

Although DP is soluble in water in the presence of Arg, it formed gel when potassium hydroxide $(\mathrm{KOH})$, instead of Arg, was mixed in a molar ratio of $\mathrm{KOH}: \mathrm{DP}=3: 1$. As $\mathrm{KOH}$ is a strong base, almost all 
of the head groups of DP might be neutralized and ionized while the tail group of the DP might be associated / aggregated due to the salting-out effect of $\mathrm{KOH}$, resulting in the gelation. On the other hand, Arg has a guanidinium group and a short hydrocarbon chain in its molecular structure, which could interact with the hydrocarbon chain of the DP. The complex between Arg and DP should have a positively charged guanidinium group and a negatively charged phosphate group as a whole. Therefore, DP might become soluble in the presence of Arg by virtue of the ionization and intermolecular interaction between them.

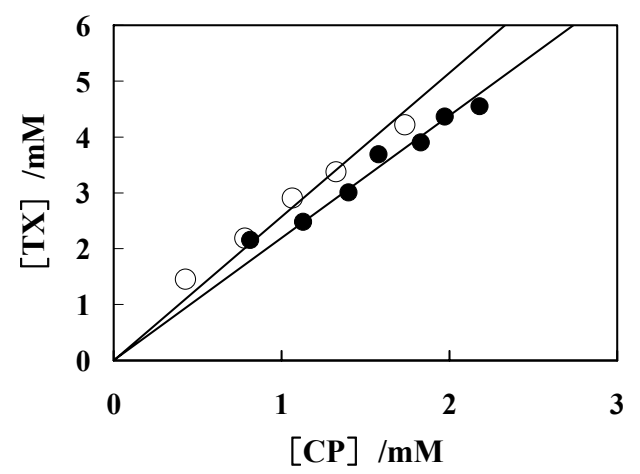

FIGURE 3 Border line between a clear solution (the upper area) and turbid one (the lower area) for the system of CP mixed with TX. Open circle is the data in the presence of Arg at a constant mixing ratio of $[\mathrm{Arg}] /[\mathrm{CP}]=3$ in mole at $30{ }^{\circ} \mathrm{C}$. Data shown by closed circle is quoted from FIGURE 2, where Arg was not added to the system of $\mathrm{CP}$ mixed with TX at $70{ }^{\circ} \mathrm{C}$.

\section{(4) Surface tension}

Surface tension of an aqueous solution of TX in the presence and absence of Arg was measured as a function of a concentration of TX in order to examine the effect of Arg on the surface activity of TX. The concentration of Arg was kept constant at 0, 0.5, and $1.5 \mathrm{mM}$ over the concentration range of TX studied, while the concentration of TX was changed from 0 to $1 \mathrm{mM}$. The results are shown in FIGURE 4. Surface tension of the pure water is shown on the ordinate $(=72.75 \mathrm{mN} / \mathrm{m})$. Critical micellization concentration (cmc) of TX in the absence of Arg was determined as $0.33 \mathrm{mM}$.
Arg alone did not exhibit remarkable surface activity. The surface tension converges on one point on the ordinate irrespective of the concentration of added Arg when the concentration of TX was zero. On the other hand, the surface tension decreased with a concentration of Arg when the concentration of TX was kept constant within the concentration range up to $0.33 \mathrm{mM}(=\mathrm{cmc})$. This fact is suggesting that the surface activity of TX was enhanced by Arg. When the concentration of TX becomes higher than the $\mathrm{cmc}$, all the curves exhibited a similar behavior regardless of the concentration of Arg added, i.e., the surface activity of TX became almost the same. The effect of TX in this concentration range becomes dominant rather than that of Arg.

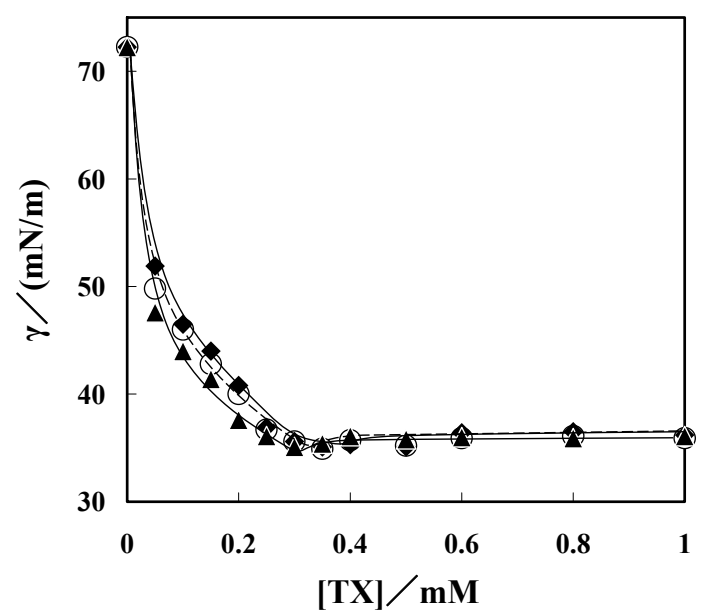

FIGURE 4 Surface tension of an aqueous solution of TX at $20{ }^{\circ} \mathrm{C}$ (room temperature) in the presence and absence of Arg as a function of a concentration of TX. The concentration of Arg added $/ \mathrm{mM}=0$ (closed diamond), 0.5 (open circle), and 1.5 (closed triangle).

Surface tension of an aqueous solution or suspension of a mixture of TX-MAP-Arg is shown as a function of a concentration of TX in FIGURE 5. Data of an aqueous solution of TX in the absence of both MAP and Arg (closed diamond) was quoted from FIGURE 4, as a reference. When $0.5 \mathrm{mM}$ DP was added to water in the absence of both Arg and TX, the DP molecule was spread over the water surface as a monolayer and showed high surface activity ${ }^{11}$. Therefore, the surface tension decreased to ca. $40 \mathrm{mN} / \mathrm{m}$, as shown on the ordinate with an open 
circle. The surface tension decreased furthermore with a concentration of TX due to their cooperative surface activity. After attaining a minimum at $c a$. 0.1 $\mathrm{mM}$ TX, the surface tension increased with the concentration and leveled off at $c a$. $0.4 \mathrm{mM} \mathrm{TX}$, where the turbid solution became almost clear. This is owing to the solubilization of DP by TX after the formation of the mixed micelle between TX and DP.

When $1.5 \mathrm{mM}$ Arg was added, the molar ratio of Arg to DP becomes $1.5 \mathrm{mM}: 0.5 \mathrm{mM}=3: 1$ and, therefore, the clear solution was obtained. Its surface tension in the absence of TX was $62 \mathrm{mN} / \mathrm{m}$, as shown by a closed circle on the ordinate. It decreased with a concentration of TX and leveled off, where the value of surface tension was almost the same as that in the absence of Arg (open circle) and also in the absence of both Arg and DP (diamond).

$0.5 \mathrm{mM} \mathrm{MP}$ and $0.5 \mathrm{mM} \mathrm{CP}$ is practically insoluble in water even $1.5 \mathrm{mM}$ Arg was added. Therefore, the surface tension of the former (open square) and the latter (closed square) was almost the same as that of the pure water $(=72.75 \mathrm{mN} / \mathrm{m})$ or that in the absence of both TX and Arg (diamond), as shown on the ordinate. It decreased with a concentration of TX and leveled off. The plateau value of the surface tension was the highest in the case of CP. It decreased in the order of CP, MP, and DP. This is the sequence of their hydrophobicity and a reverse order of their solubility, as mentioned before.

The precipitate remaining undissolved does not contribute to the surface tension, but it adsorbs the TX dissolved in an aqueous phase. The precipitate might be swelled in some degree, forming the blob or swelled aggregate. The adsorption or consumption of TX from the aqueous phase was accelerated through the intermolecular hydrophobic interaction on/in the blob. The consumption of TX from the aqueous phase induces also the concomitant desorption of TX from the air/water interface in order to newly attain the equilibrium state after the disturbance. Thus, the surface tension increases after the consumption of TX by the blob. The order of CP, MP, and DP observed in FIGURE 5 was explained in the three terms, i.e., solubility of the MAP, adsorption of the TX to the blob, and concurrent desorption of TX from the interface.

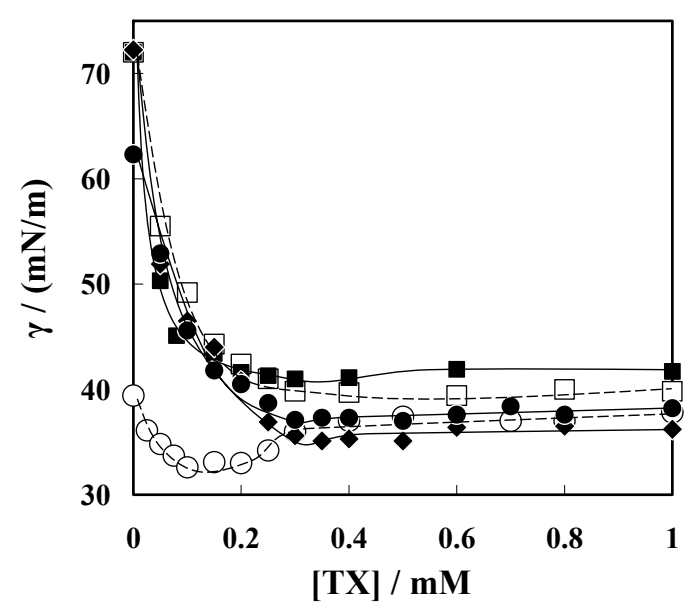

FIGURE 5 Surface tension of a mixture of TX-Arg-MAP in water as a function of a concentration of TX at $20{ }^{\circ} \mathrm{C}$. Closed diamond: TX alone (the data was quoted from FIGURE 4), open circle: TX $+0.5 \mathrm{mM}$ DP in the absence of Arg, closed circle: $\mathrm{TX}+1.5 \mathrm{mM}$ Arg $+0.5 \mathrm{mM} \mathrm{DP}$, open square: $\mathrm{TX}+1.5 \mathrm{mM}$ Arg $+0.5 \mathrm{mM} \mathrm{MP}$, and closed square: $\mathrm{TX}+1.5 \mathrm{mM}$ Arg $+0.5 \mathrm{mM} \mathrm{CP}$.

\section{(5) Solubility of methyl yellow}

Methyl yellow (MY) is practically insoluble in water. It is known that mixed micelle of TX-DP in an aqueous phase entraps MY by virtue of hydrophobic interaction $^{12,13}$. The solubility of MY was studied in the presence of the mixed micelle of MAP-TX-Arg. The results are shown in FIGURE 6. Data for MP-TX-Arg was omitted to avoid confusion due to the crowd in the figure. As the concentration range of TX shown in FIGURE 6 is broader than that in FIGURE 5, $\mathrm{CP}$ as well as MP and DP was clearly dissolved in the presence of TX and Arg. This fact could be confirmed from the data shown in FIGURE 3, where the slope of $[\mathrm{TX}] /[\mathrm{CP}]$ in the presence of Arg is approximately 2.5. Therefore, the data for $\mathrm{CP}$ in FIGURE 6 was obtained in the concentration range of TX higher than $2.5 \mathrm{mM}$, as shown by closed circle. It was also confirmed that Arg alone does not affect the solubility of MY.

The arrow mark in FIGURE 6 shows the cmc of TX $(=0.33 \mathrm{mM})$. The solubility increased with a concentration of TX when it was higher than the cmc. Triangle on the ordinate shows the solubility of MY 
in water $(=c a .0 .01 \mathrm{mM})$. The slope becomes steeper in the presence of $1 \mathrm{mM}$ MAP, of which sequence was DP, MP, and CP. This is because the mixed micelle becomes larger and the capacity for MY also increases with the chain length. It was confirmed that the slope increases with a concentration of a MAP added (data not shown here).

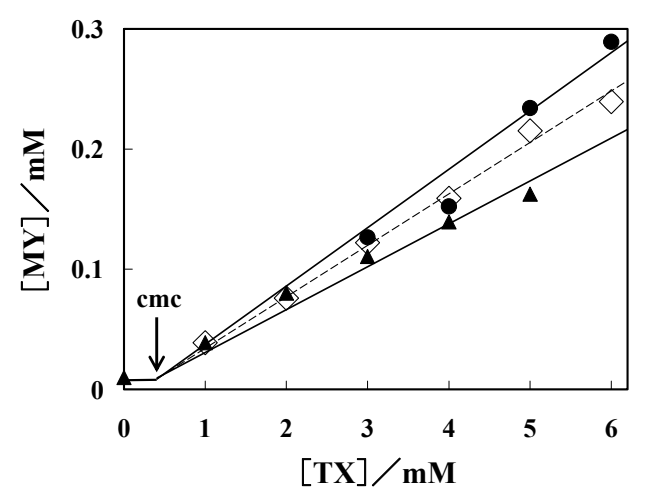

FIGURE 6 Solubility of MY as a function of a concentration of added TX in the presence of $1 \mathrm{mM}$ MAP and $3 \mathrm{mM}$ Arg at $30{ }^{\circ} \mathrm{C}$. The mixing molar ratio of Arg to MAP was kept constant at 3:1. Closed triangle: TX alone, open diamond: $\mathrm{TX}+3 \mathrm{mM} \mathrm{Arg}+$ $1 \mathrm{mM}$ DP, closed circle: TX $+3 \mathrm{mM}$ Arg $+1 \mathrm{mM} \mathrm{CP}$.

\section{CONCLUSION}

Although MAP is almost insoluble in water at room temperature, it was solubilized in the presence of Triton at $70{ }^{\circ} \mathrm{C}$. DCP was practically insoluble even at $70{ }^{\circ} \mathrm{C}$. However, the MAP became soluble at $30{ }^{\circ} \mathrm{C}$ in the presence of Arg together with some amount of Triton. Basic Arg accelerated ionization and neutralization of an acidic head group of the MAP, while Triton formed mixed micelle with the MAP. According to the surface tension data, the MAP ionized by Arg showed higher affinity for water than the untreated MAP of nonionic type. Mixed micelle of TX and MAP solubilized MY, where the solubilizing power became stronger with the chain length and contents of the MAP in the mixed micelle when the concentration of TX is kept constant.

\section{REFERENCES}

1. S. Shimabayashi, K. Furukawa, and T. Hino, in Advances in Crystal Growth Inhibition Technologies, Edited by Zhahid Amjad (Kluwer Academic/Plenum Publishers, New York, 2000), Chap. 4, pp. 43-55.

2. H.Tanaka, A. Yasuoka, K. Kandori, and T. Ishikawa, Langmuir, 13, 821(1997).

3. T. Ishikawa, H. Tanaka, A. Yasuoka, and K. Kandori, J. Mater.Chem., 5, 1963(1995).

4. H.Tanaka, T. Watanabe, M. Chikazawa, and K. Kandori,, Colloids Surfaces A, 139, 342 (1998).

5. H. Tanaka, A. Yasukawa., K. Kandori, and T. Ishikawa, Colloids Surfaces A, 125, 53(1997).

6. K. Warabino and S. Shimabayashi, Phos. Res. Bull., 19, 294(2005).

7. K. Warabino, S. Ueno, T.Hino, and S. Shimabayashi, Phos. Res. Bull., 20, 129(2006).

8. N. Oda and S. Shimabayashi, Phos. Res. Bull., 19, 198(2005).

9. K. Nakayama, I. Tari, M. Sakai, Y. Murata, and G. Sugihara, J. Oleo Sci., 53, 247(2004).

10. T. Inoue, N. Nakashima, G. Sugihara, and Y. Yoshino, J. Oleo Sci., 54, 95 (2005).

11. M. Hossain, T. Suzuki, and T. Kato, J. Colloid Interface Sci., 288, 342 (2005).

12. S. Shimabayashi, T. Hino, and T. Ohnishi, Phos. Res. Bull., 11, 31(2000).

13. S. Shimabayashi and T. Hino, Phos. Res. Bull., 13, 21(2002). 